\title{
Study of Molecular Arrangement and Surface Morphology of Organized Molecular Films of Charge-Transfer Complexes Containing Metal(dmit) $)_{2}$ Unit
}

\author{
Ryosuke Itagaki ${ }^{1}$, Yoko Tatewaki ${ }^{2}$, and Atsuhiro Fujimori ${ }^{2} *$ \\ ${ }^{1}$ Department of Polymer Science and Engineering, Faculty of Engineering, ${ }^{2}$ Graduate School of Science and Engineering, \\ Yamagata University, Yonazawa, Yamagata, 992-8510, Japan \\ Fax:+81-238-26-3073, e-mail: fujimori@yz.yamagata-u.ac.jp
}

The molecular arrangement and surface morphology of organized molecular films of alkylammonium-M bis(1, 3-dithiole-2-thione-4, 5-dithiolate) $\left((\mathrm{dmit})_{2}, \mathrm{M}=\mathrm{Ni}, \mathrm{Au}\right.$, and $\left.\mathrm{Pd}\right)$ charge-transfer complexes were investigated by using the surface pressure-area isotherm, in-plane and out-of plane X-ray diffractions (XRD), and atomic force microscopy. In the bulk state, several alkylammonium-M(dmit) ${ }_{2}$ molecules could not form a highly ordered layer structure along the $c$-axis and a sub-cell structure of the alkyl chain in the $a b$-plane; however, almost all molecules formed a layer structure in the film multilayers. Monolayers of alkylammonium-M(dmit $)_{2}$ salts on the water surface were relatively condensed. Out-of plane and in-plane XRD measurements revealed that over a long period, systematic changes occurred in the two-dimensional lattice of alkylammonium-M(dmit $)_{2}$ salts and not in their bulk state. These structural changes appear to be caused by enhancement of the van der Waals interaction among long hydrocarbons and the $\pi-\pi$ interaction among $\mathrm{M}(\mathrm{dmit})_{2}$ units arranged two-dimensionally. In addition, both the molecular arrangement and the morphology of the films showed dependence on the hydrocarbon length, number of alkyl chains, and kind of central metal. In particular, the molecular arrangement of materials having didecyl chains changed drastically and $\mathrm{M}(\mathrm{dmit})_{2}$ units were highly oriented in the $a b$-plane.

Key words: alkylammonium-metal(dmit $)_{2}$; layer structure; two-dimensional lattice; molecular arrangement; surface morphology

\section{INTRODUCTION}

Alkylammonium-metal bis(1, 3-dithiole-2thione-4, 5-dithiolate $\left(\mathrm{M}(\mathrm{dmit})_{2}\right)$ complexes exhibit a variety of electrical properties ranging from dielectric to superconducting. ${ }^{1}$ Langmuir-Blodgett (LB) films of dialkylammonium-Au(dmit) $)_{2}$ salts exhibited a high lateral conductivity of $40-100 \mathrm{~S} / \mathrm{cm}$ at room temperature after electrochemical oxidation or $\mathrm{I}_{2}$ doping; these films also showed metallic temperature dependence of the conductivity. ${ }^{2}$ Furthermore, the ac magnetic susceptibility and resistance of these films suggested the existence of a superconducting phase below $4 \mathrm{~K}^{3}$ Nakamura et al. conducted pioneering studies on structural and conductive properties of LB films containing $\mathrm{M}(\mathrm{dmit})_{2}$ with various central metals. ${ }^{4}$ Miura and Sugi conducted extensive research on conductive LB films of charge-transfer complexes containing dimt. ${ }^{2,3,5}$

It has been elucidated that field-effect doping (FE doping) is an effective carrier doping method for organic semiconductors. An important requirement for achieving optimum transport characteristics is to construct a well-ordered conducting path for charge carriers, along which oriented $\pi$-conjugated molecular systems interact with each other in the face-to-face direction to form a stacking structure. ${ }^{6}$ The LB technique is useful in controlling the molecular arrangements and in constructing the conducting path for charge carriers. ${ }^{7,8}$

In a previous study, we attempted to improve conductivities of an LB film containing alkylammonium-M(dmit $)_{2}$ complexes by using the FE doping method. ${ }^{9}$ If the mechanism of conduction of this LB film is elucidated, we can use the obtained information to fabricate novel devices. For driving the functionality of these organic molecular devices in an efficient manner, it is a prerequisite to control the molecular orientation and arrangement at the monomolecular level. However, very few studies have carried out a detailed structural estimation and morphological observation of organized molecular films of alkylammonium-M(dmit $)_{2}$ on the sub-nanometer scale, whereas several studies have extensively investigated conductive properties of LB films ${ }^{46}$ and carried out a structural estimation of three-dimensional single crystals..$^{5,10}$

By the way, functional molecular assemblies, which are precisely controlled molecular arrangements, and their organized molecular films ${ }^{11}$ can be developed as potential candidates for biomimetic models ${ }^{12}$ and molecular electronic devices $^{7}$, which have attracted considerable interest in basic science; further, these molecular assemblies have several potential applications.

It is commonly known that long-chain compounds, which have crystallizable long hydrophobic chains attached to functional groups, are packed into a layer structure ${ }^{13}$ along the $c$-axis and sub-cell of the long chains in the $a b$-plane. ${ }^{14}$ This "sub-cell" structure ${ }^{15}$ of the n-alkyl chain in amphiphiles is formed as a two-dimensional lattice, and the "layer structure" along the $c$-axis often reflects the large spacing between hydrophobic chains in accumulated double-layer structures.

In this study, we investigate the molecular orientation and surface morphology of organized molecular films of alkylammonium-M(dmit) $)_{2}(\mathrm{M}$ $=\mathrm{Ni}, \mathrm{Au}$, and $\mathrm{Pd}$ ) salts on a solid by the surface 
pressure-area $(\pi-\mathrm{A})$ isotherm, polarized visible spectroscopy, in-plane and out-of plane X-ray diffractions (XRD), and atomic force microscopy (AFM). We show that it is possible to accurately control the molecular arrangement, packing, and regularity of the layered organization by methods of formation of organized molecular films. ${ }^{16}$

\section{EXPERIMENTAL}

\subsection{Materials}

Alkylammonium- $\mathrm{M}(\mathrm{dmit})_{2}$ salts (abbrev. $\left(\mathrm{C}_{\mathrm{n}} \mathrm{H}_{2 \mathrm{n}+1}\right)_{\mathrm{m}} \mathrm{N}\left(\mathrm{CH}_{3}\right)_{4-\mathrm{m}}-\mathrm{M}(\mathrm{dmit})_{2},(\mathrm{M}=\mathrm{Ni}, \mathrm{Au}$, and $\mathrm{Pd})$, Fig. 1) were synthesized using the same procedure as that described in previous studies. ${ }^{9}$ In this study, monolayers of $\left(\mathrm{C}_{\mathrm{n}} \mathrm{H}_{2 \mathrm{n}+1}\right)_{\mathrm{m}} \mathrm{N}\left(\mathrm{CH}_{3}\right)_{4-\mathrm{m}}-\mathrm{M}(\mathrm{dmit})_{2}$ were spread from acetonitrile/benzene $(50 / 50, \mathrm{v} / \mathrm{v})$ mixed solutions (approximately $10^{-4} \mathrm{M}$ ) onto distilled water (18 $\mathrm{M} \Omega \cdot \mathrm{cm})$. The $\pi-\mathrm{A}$ isotherms of the monolayers were measured by a FACE film balance (Kyowa Kaimen Co.) at $17{ }^{\circ} \mathrm{C}$. Then, these monolayers were transferred onto glass (XRD samples) or mica (AFM samples) substrates by the horizontal lifting method at various surface pressures in order to obtain nonalternating X-type films.

\subsection{Characterization}

The layer structures in organized molecular films were characterized by out-of plane XRD using a RAD-rA diffractometer (Rigaku Co.). Monochromatized $\mathrm{CuK} \alpha$ radiation was generated at $40 \mathrm{kV}$ and $100 \mathrm{~mA}$. The diffracted X-ray beam was monochromatized by a pyrographite monochromatic system and monitored by a scintillation counter. The in-plane spacing of the two-dimensional lattice of the films was determined by analysis using an X-ray diffractometer (MXP-BX, Bruker AXS; $\mathrm{CuK \alpha}$ radiation, $40 \mathrm{kV}, \quad 40 \mathrm{~mA}$, custom-made instrument); the diffractometer was equipped with a parabolic graded multilayer mirror. The surface morphologies of the films were observed by a tapping mode scanning probe microscope (SPA300 with an SPI3800 probe station, SII) using microfabricated $\mathrm{Si}$ cantilevers with integrated tips with a constant force of $1.4 \mathrm{~N} / \mathrm{m}$.

\section{RESULTS AND DISCUSSION}

From the results of $\pi-\mathrm{A}$ isotherms, monolayers of didococyldimethylammonium-Ni(dmit $)_{2}$ (abbrev. 2(C22)-Ni), dioctadecyldimethylammonium-Ni(dmit) ${ }_{2} \quad$ (2(C18)-Ni), didecyldimethylammonium- $\mathrm{Au}(\mathrm{dmit})_{2} \quad$ (2(C10)Au), and tridecylmethylammonium-Ni(dmit $)_{2}$ (3(C10)-Ni) salts formed liquid expand monolayers. As compared to these four monolayers, monolayers of didodecyl-

$$
\begin{aligned}
& \left(\mathrm{C}_{\mathbf{n}} \mathrm{H}_{2 \mathrm{n}+1}\right)_{\mathbf{m}} \mathrm{N}^{+}\left(\mathrm{CH}_{3}\right)_{4-\mathbf{m}}(\mathrm{S}=22,18,14,12,10) \\
& \mathrm{m}=2,3,4 \\
& \mathrm{M}=\mathrm{Ni}, \mathrm{Au} \text {, and } \mathrm{Pd}
\end{aligned}
$$

Fig. 1 Chemical structure of alkylammonium $\mathrm{M}(\mathrm{dmit})_{2}(\mathrm{M}=\mathrm{Ni}, \mathrm{Au}$, and $\mathrm{Pd})$.
dimethylammonium-Ni(dmit $)_{2}$ ditetradecyldimethylammonium-Ni(dmit $)_{2}$ (2(C14)-Ni), and didecyldimethylammonium$\mathrm{Ni}(\mathrm{dmit})_{2}$ (2(C10)-Ni) show relatively lower collapsed surface pressures. The monolayer of the tetradecylmethylammonium-Ni(dmit $)_{2}$ (4(C10)-Ni) salt collapsed at approximately 5 $\mathrm{mNm}^{-1}$ and showed a wide molecular area of over $40 \AA^{2} /$ molecule. The dioctadecyldimethylammonium-Pd(dmit $)_{2}$ (2(C18)-Pd) salt extremely expanded on the water surface.

Figure 2 shows out-of plane XRD profiles of X-type multilayers (15 layers) of several alkylammonium-M(dmit $)_{2}$ salts. In the case of estimation for the dependency on chain-lengths, X-type multilayers of 2(C22)-Ni, 2(C18)-Ni, 2(C14)-Ni, 2(C12)-Ni, and 2(C10)-Ni salts exhibit the systematically changes in long spacing. Figure 2(b) shows plots of number of $\mathrm{C}$ atoms in the alkyl chain versus long spacing and estimated molecular length of only dialkyl ammonium by a simulation. A simple speculation is that the difference between the calculated value of molecular length and long spacing corresponds to $\mathrm{M}(\mathrm{dmit})_{2}$ unit itself. Needless to say, the tilt angle of the alkyl chain cannot be ignored. However, if we assume the normal orientation of the alkylammonium part in this case, the values of the abovementioned difference corresponding to the $\mathrm{M}(\mathrm{dmit})_{2}$ unit are $2.6(\mathrm{C} 22), 3.5$ (C18), 5.4 (C14), 5.2 (C12), and 4.9 (C10) Å, respectively. These values are relatively smaller than those of the normal-oriented end-on type $\mathrm{M}(\mathrm{dmit})_{2}$ unit.

In the case of comparison with number of alkyl chains, systematic changes in long spacing are also confirmed and multilayers of $3(\mathrm{C} 10)-\mathrm{Ni}$ show two kinds of layer spacing. Further, (a)
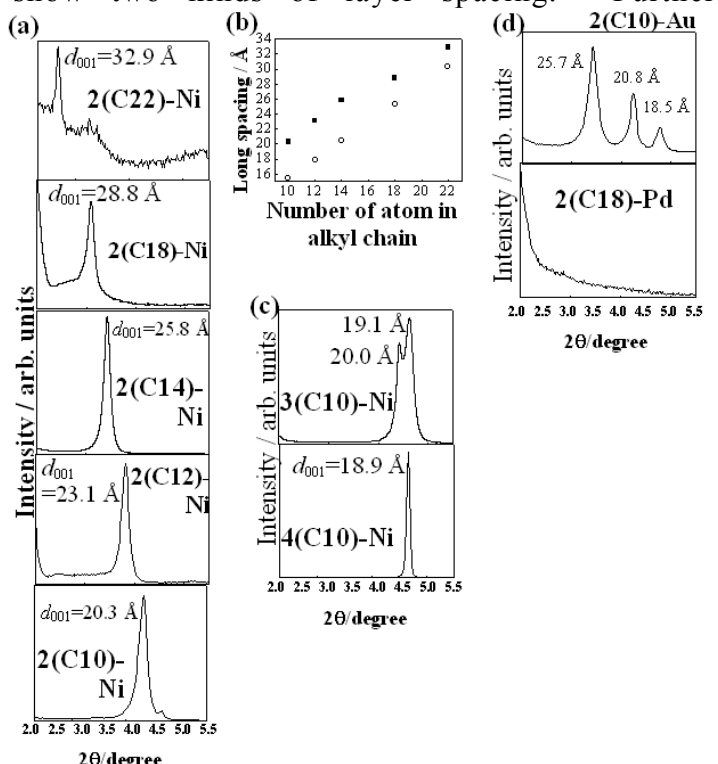

Fig. 2 Out-of plane XRD profiles of multilayers of $\left(\mathrm{C}_{\mathrm{n}} \mathrm{H}_{2 \mathrm{n}+1}\right)_{\mathrm{m}} \mathrm{N}\left(\mathrm{CH}_{3}\right)_{4-\mathrm{m}}-\mathrm{M}(\mathrm{dmit})_{2}$ salts transferred by horizontal lifting method; (a) dependency on chain length and (b) plots of number of $\mathrm{C}$ atoms in alkyl-chain vs. -long spacing and oestimated molecular length of dialkyl ammonium by calculation, (c) dependency on number of chains, and (d) dependency on central metals. 
multilayers of 2(C10)-Au show three layer spacings, and 2(C18)-Ni, which forms an expanded monolayer on water surface, is unable to form the layer structure along the $c$-axis.

In order to evaluate the ability of alkylammonium-M(dmit $)_{2}$ salts in bulk to form a layer structure, WAXD measurements were carried out. As a result, formation of a highly ordered layer structure along the $c$-axis is not essentially a stable feature of these charge-transfer complexes in bulk. Almost all complexes formed a peculiar two-dimensional arrangement and showed layered organization by method of organized molecular films.

Figure 3 shows in-plane XRD profiles of X-type multilayers of several alkylammonium$\mathrm{M}(\mathrm{dmit})_{2}$ salts. Multilayers of $2(\mathrm{C} 22)-\mathrm{Ni}$ and 2(C18)-Ni show relatively weak signals at $4.1 \AA$, whereas multilayers of $2(\mathrm{C} 14)-\mathrm{Ni}$ and $2(\mathrm{C} 12)-\mathrm{Ni}$ show a halo and low-intensity peak at $6.8 \AA$. The in-plane XRD profiles of the salts show that the molecular arrangement of 2(C10)-Ni changes drastically with a change in the chain length. Five different in-plane spacings-8.7, 6.8, 5.2, 4.4 , and $4.1 \AA$-are clearly confirmed in multilayer of this salt.

The 2(C10)-Au multilayers also show five two-dimensional spacings. It is supposed that five in-plane spacings for the 2(C10)-Au multilayers also contain distance originated from alkyl-chain packing and another factors. The multilayers of 2(C22)-Ni, 2(C18)-Ni, 3(C10)-Ni, and $4(\mathrm{C} 10)-\mathrm{Ni}$ have two-dimensional hexagonal packing. The lattice spacing of $2(\mathrm{C} 22)-\mathrm{Ni}$, $2(\mathrm{C} 18)-\mathrm{Ni}$, and $4(\mathrm{C} 10)-\mathrm{Ni}$ is $4.1 \AA$, whereas that of $3(\mathrm{C} 10)-\mathrm{Ni}$ is $5.3 \AA$. On the other hand, 2(C10)-Ni has orthorhombic packing at lattice spacings of 5.2 and $4.1 \AA$ in its multilayers. In the two-dimensional plane, hexagonal systems are essentially similar to orthorhombic ones, though their unit cells are different. The $2(\mathrm{C} 10)-\mathrm{Ni}$ is speculated to have a subtly distorted two-dimensional lattice in the film. The most distorted two-dimensional lattice is observed in

(a)

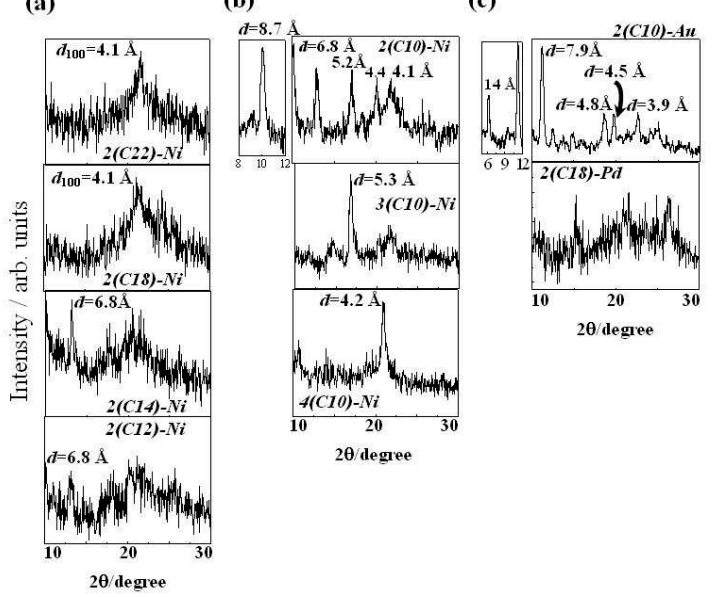

Fig. 3 In-plane XRD profiles of multilayers of $\left(\mathrm{C}_{\mathrm{n}} \mathrm{H}_{2 \mathrm{n}+1}\right)_{\mathrm{m}} \mathrm{N}\left(\mathrm{CH}_{3}\right)_{4-\mathrm{m}}-\mathrm{M}(\mathrm{dmit})_{2}$ salts transferred by horizontal lifting method; (a) dependency on chain length, (b) dependency on number of chains, and (c) dependency on central metals. the film of the 2(C10)-Au. This salt has triclinic packing with three different lattice spacings for the hydrocarbon chains.

Figures 4 show schematic illustrations of molecular arrangements of organized molecular films of 2(C10)-Ni and 2(C10)-Au salts, respectively. In a previous study, it was found that the multilayers of both $2(\mathrm{C} 10)-\mathrm{Ni}$ and 2(C10)-Au salts exhibited properties of an active field effect transistor (FET) ${ }^{9}$; therefore, closely packed $\mathrm{M}(\mathrm{dmit})_{2}$ units were expected to be formed in the films of these salts. From the results of in-plane XRD measurements, characteristic in-plane spacing was confirmed in the profiles of these materials. In the case of $2(\mathrm{C} 10)-\mathrm{Ni}$, we concluded that the in-plane spacings of 5.2 and $4.1 \AA$ corresponded to the in-plane spacing formed by the alkyl chain judging from the results of $3(\mathrm{C} 10)-\mathrm{Ni}$ and $4(\mathrm{C} 10)-\mathrm{Ni}$ salts. Within the residual three peaks at $8.7,6.8$, and $4.4 \AA$, spacing of 8.7 and $4.4 \AA$ probably correspond to the first and second order reflections. This highly ordered structure with a spacing of $8.7 \AA$ was considered to be the repeating unit of the two-dimensional $\mathrm{M}(\mathrm{dmit})_{2}$ arrangement. By a simple calculation, the length of the $\mathrm{M}(\mathrm{dmit})_{2}$ unit along the long axis was determined to be approximately $17 \AA$; therefore, a two-dimensional tilted arrangement was assumed to be formed in the film of 2(C10)-Ni. In this case, tilt angle from the molecular plane was $51^{\circ}$. On the other hand, the

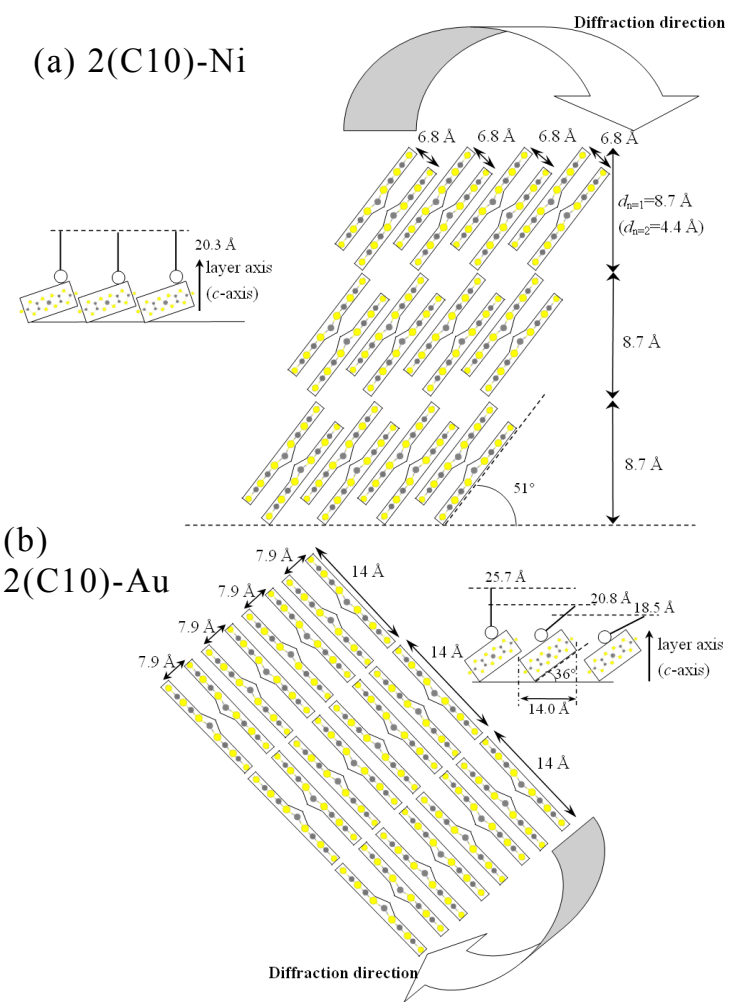

Fig. 4 Models of two-dimensional arrangements of (dmit) $)_{2}$ units in organized molecular films and schematic illustration of layer alignment along the $c$-axis of (a) $\left(\mathrm{C}_{10} \mathrm{H}_{21}\right)_{2} \mathrm{~N}\left(\mathrm{CH}_{3}\right)_{2}-\mathrm{Ni}(\mathrm{dmit})_{2}$ and $\left(\mathrm{C}_{10} \mathrm{H}_{21}\right)_{2} \mathrm{~N}\left(\mathrm{CH}_{3}\right)_{2}-\mathrm{Au}(\mathrm{dmit})_{2}$ salts. (b) 
spacing of $6.8 \AA$ was the distance between $\mathrm{M}(\mathrm{dmit})_{2}$ units facing each other. This spacing was also confirmed in the in-plane XRD profiles of 2(C14)-Ni and 2(C12)-Ni. Although the $\mathrm{M}(\mathrm{dmit})_{2}$ unit is almost flat-type group, gap from the molecular plane is caused by changes in the stable configuration with a change in the radius of the covalent bond of the central metals. This model of molecular arrangement is probably accurate because the absence of highly ordered molecular pairs cannot yield two-dimensional repeating units.

Figure 4(b) shows a model of characteristic molecular orientation of $2(\mathrm{C} 10)-\mathrm{Au}$ multilayers. In this case, we concluded that the in-plane spacings of $4.8,4.5$, and $3.9 \AA$ correspond to that in the triclinic sub-cell of hydrocarbons. The spacings of 14.0 and $7.9 \AA$ shown in this figure are the distances between $\mathrm{M}(\mathrm{dmit})_{2}$ units in the film plane. As mentioned previously, it is supposed that gap from the molecular plane is caused by changes in the stable configuration with a change in the radius of the covalent bond of the central Au. In this case, the distance of $7.9 \AA$ corresponds to the spacing between $\mathrm{M}(\mathrm{dmit})_{2}$ units facing each other in the complex containing $\mathrm{Au}$. Since the radius of the $\mathrm{Au}$ is larger than that of the $\mathrm{Ni}$ atom, it is supposed that the distortion of the molecular plane is relatively large and that the distance between (dimt) $)_{2}$ pairs is large. The residual spacing of $14 \AA$ is the two-dimensional repeating unit of coupled $\mathrm{M}(\mathrm{dmit})_{2}$ units facing each other. The spacing of $14 \AA$ is relatively large, and from the results of polarized visible spectra, it is unlikely that the

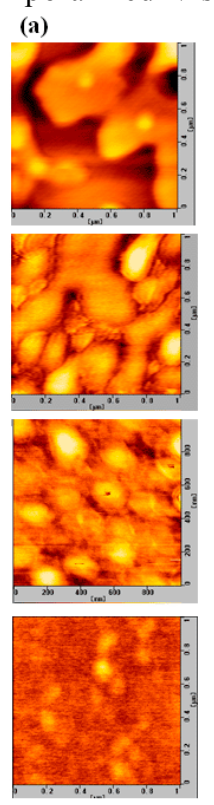

Fig. 5 Tapping-mode AFM images $\left(1 \times 1 \mu \mathrm{m}^{2}\right)$ of several $X$-type monolayers on the mica substrate of $\left(\mathrm{C}_{\mathrm{n}} \mathrm{H}_{2 \mathrm{n}+1}\right)_{\mathrm{m}} \mathrm{N}\left(\mathrm{CH}_{3}\right)_{4-\mathrm{m}}-\mathrm{M}(\mathrm{dmit})_{2}$ salts; (a) dependency on chain length; 2(C22)-Ni, 2(C18)-Ni, 2(C14)-Ni, and 2(C12)-Ni (from top to down), (b) dependency on number of chains; 2(C10)-Ni, 3(C10)-Ni, and $4(\mathrm{C} 10)-\mathrm{Ni}$ (from top to down), and (c) dependency on central metals; $2(\mathrm{C} 10)-\mathrm{Au}$, and 2(C18)-Pd (from top to down).
$\mathrm{M}(\mathrm{dmit})_{2}$ unit is arranged at a highly tilted angle with respect to the surface normal. Therefore, it is inferred that a tilt angle of $36^{\circ}$ to the solid surface, which corresponds to an in-plane spacing of $14.0 \AA$, is suitable.

Figure 5 shows AFM images of X-type monolayers for several alkylammonium-M(dmit $)_{2}$ salts. The monolayer of 2(C22)-Ni shows both shapeless and small circular domains. The monolayer of 2(C18)-Ni shows domains that are partially combined with many circular domains. Further, the monolayer of $2(\mathrm{C} 14)-\mathrm{Ni}$ shows isolated circular domains with a diameter of 200 $\mathrm{nm}$. The monolayers of 2(C12)-Ni salts, which have randomly oriented $\mathrm{M}(\mathrm{dmit})_{2}$ units, as confirmed by polarized visible spectra, show rather small and unclear domains. However, in the case of monolayer of $2(\mathrm{C} 10)-\mathrm{Ni}$, there are no systematic changes in domain size with a change in the chain length, and both large and small domains with diameters in the range of $200 \mathrm{~nm}$ to $50 \mathrm{~nm}$ are present. That is, it is possible to control the size and shape of mesoscopic domains on the monolayer surface of alkylammonium-M(dmit $)_{2}$ salts by changing the chain length, number of hydrophobic chains, and central metal.

\section{ACKNOWLEDGEMENTS}

One of the authors (Y. T.) sincerely thanks Prof. T. Nakamura of Research Institute for Electronic Science, Hokkaido Univ. and Prof. K. Ikegami of the Nanotechnology Research Institute, AIST, for his kind collaboration.

\section{REFERENCES}

[1] "Organic Superconductors" Ed. By T. Ishiguro, K. Yamaji, G. Saito, Springer Series in Solid-State Sciences, Springer, Berlin, 1977.

[2] Y. F. Miura, Y. Okumo, H. Ohnishi, T. Kawasaki, M. Sugi, Jpn. J. Appl. Phys., 1998, 37, 1481.

[3] Y. F. Miura, M. Horikiri, S. Tajima, T. Wakaita, S. -H. Saito, M. Sugi, Synth. Met., 2003, 133-134, 663.

[4] T. Nakamura, K. Kojima, M. Matsumoto, H. Tachibana, M. Tanaka, E. Manda, Y. Kawabata, Chem. Lett., 1989, 367.

[5] Y. F. Miura, M. Horikiri, K. Honda, M. Shiro, K. Sasaki, M. Sugi, Jpn. J. Appl. Phys., 2004, 43, 2628. [6] H. Kobayashi, R. Kato, T. Mori, A. Kobayashi, Y. Sasaki, G. Saito, T. Enoki, H. Inokuchi, Mol. Cryst. Liq. Cryst., 1984, 107, 33.

[7] G. G. Roberts, "Langmuir-Blodgett Films", Plenum Press, London, 1990, M. C. Petty, "Langmurr-Blodgett Films" Cambridge Univ. Press, New York, 1996.

[8] M. R. Bryce, M. C. Petty, Nature, 1995, 374, 771. [9] Y. Tatewaki, H. Ohnuki, K. Ikegami, Trans., Mater. Res. Soc. Jpn, 2006, 32, 345.

[10] W. S. Xia, C. H. Huang, D. J. Zhou, Langmuir, $1997,13,80$.

[11] G. L. Gaines, Jr., "Insoluble Monolayers at Liquid Gas Interfaces", Wiley, New York, 1966.

[12] H. Kuhn, D. Möbius, H. Bucher, Spectroscopy of Monolayer Assemblies, in "Physical Methods of Chemistry" (A. Weissberger, B. W. Rossiter, Eds.), Vol. 1, Part IIIB, pp. 577-702, Wiley, New York, 1972 .

[13] A. Keller, Phil. Mag., 1957, 2, 1171.

[14] K. Inomata, Y. Sakamaki, T. Nose, S. Sasaki, Polym. J., 1996, $28,992$.

[15] V. Vand, Acta Cryst., 1951, 4, 465

[16] Y. Tatewaki, S. Okada, R. Itagaki, T. Nakamura, A. Fujimori, J. Colloid Interf. Sci., 2010, $343,281$. 